\title{
Association of Mycobacterium avium subsp. paratuberculosis and SLC11A1 polymorphisms in Sardinian multiple sclerosis patients
}

\author{
Davide Cossu ${ }^{1}$, Speranza Masala ${ }^{1}$, Eleonora Cocco $^{2}$, Daniela Paccagnini ${ }^{1}$, Stefania Tranquilli ${ }^{2}$, \\ Jessica Frau ${ }^{2}$, Maria Giovanna Marrosu², Leonardo Antonio Sechi ${ }^{1}$ \\ ${ }^{1}$ Dipartimento di Scienze Biomediche, Sezione di Microbiologia Sperimentale e Clinica, Università di Sassari, Italy \\ ${ }^{2}$ Centro Sclerosi Multipla, Dipartimento di Scienze Cardiovascolari e Neurologiche, Università di Cagliari, Italy
}

\begin{abstract}
Introduction: Recent findings propose that Mycobacterium avium subsp. paratuberculosis (MAP) infection could act as risk factor in favoring multiple sclerosis (MS) progression. SLC11A1 is a gene associated with mycobacterial survival in the host and it may be involved in the induction and maintenance of autoimmune disease.

Methodology: In this preliminary study, $100 \mathrm{MS}$ patients and 100 healthy controls (HCs) from Sardinia were enrolled. Eight single nucleotide polymorphisms (SNPs) in the SLC11A gene were searched by PCR RFLP-genotyping. IS900 specie specific PCR was undertaken to search for MAP presence. Indirect ELISA was performed to asses if MS patients displayed a stronger humoral response against MAP2694 protein compared to the HCs.

Results: Only rs2276631 SNP was associated with MS. MAP DNA was detected in 23 out of 100 MS patients (23\%) and in 7 out of 100 HCs (7\%). A strong humoral response against MAP2694 protein was detected in 36\% of MS patients and only in 3\% of HCs. A correlation between ELISA sero-positivity and the rs2276631 SNP was also found.

Conclusion: Our preliminary results suggest that the Sardinian population might be prone to develop autoimmune disease due to polymorphisms in immunomodulating the $S L C 11 A 1$ gene, which is important in the immune response against intracellular bacteria such as MAP.
\end{abstract}

Key words: Mycobacterium avium subsp. paratuberculosis; SLC11A1; multiple sclerosis; Sardinia

J Infect Dev Ctries 2013; 7(3):203-207.

(Received 10 May 2012 - Accepted 09 July 2012)

Copyright (C) 2013 Cossu et al. This is an open-access article distributed under the Creative Commons Attribution License, which permits unrestricted use, distribution, and reproduction in any medium, provided the original work is properly cited.

\section{Introduction}

Multiple sclerosis (MS) is a complex multifactorial autoimmune disease with unknown etiology not imputable to a single genetic or environmental factor. We recently demonstrated that Mycobacterium avium subsp. paratuberculosis (MAP) is an environmental agent associated with MS in the Sardinian population $[1,2]$. The rate of MS in Sardinia is one of the highest worldwide [3], probably due to the particular genetic structure of Sardinian people. SLC11A1 is located in chromosome $2 \mathrm{q} 35$ and encodes the natural resistance associated macrophage protein 1 (NRAMP1). This protein is a divalent transition metal transporter that regulates macrophage activation in infectious and autoimmune diseases; it also exerts a role in the resistance to intracellular pathogens including mycobacteria [4]. Recently it has been associated with human Mycobacterium avium complex infection [5].

We conducted an ongoing study aiming to understand in which way genes and environment interact with each other. We have genotyped 8 SLC11A1 polymorphisms, aiming to establish if they could be associated with MAP presence. We are currently searching for MAP DNA within peripheral blood mononuclear cells (PBMCs) of MS patients and healthy controls (HCs). MAP specific antibody (Abs) detection directed against MAP2694 recombinant antigen was also confirmed. Logistic regression analysis revealed an interesting correlation between Abs presence and the genotype AA at rs2276631 SNP. These are preliminary findings that need to be confirmed in a larger study.

\section{Methodology}

Study population

This opening study included 100 MS patients and 100 HCs. Blood samples were collected as described elsewhere [1] and all of them were evaluated blindly.

MS patients were diagnosed according to the 2010 Revised McDonald criteria [6]. All of them were 
Sardinian. Seventy were female and 30 were male with an $\mathrm{F} / \mathrm{M}$ ratio of 2.3/1. Age at inclusion $=40(\mathrm{SD}$ $\pm 10.8)$, age at onset $=31(\mathrm{SD} \pm 10.8)$, and disease duration from onset $=12(\mathrm{SD} \pm 9.7)$. The clinical course was relapsing remitting in $82 \%$, secondary progressive in $12.5 \%$, and primary progressive in $5.5 \%$. The neurological disability evaluated with the EDSS score was a mean of $2(\mathrm{SD} \pm 2)$ and ranged from 0 to 6.5 .

HCs were all ethnically Sardinian for at least three generations; 65 were females and 35 were males (F/M: 1.9/1). The mean of actual age was 46 years. Subjects with no personal or familial (first-degree relatives) history of autoimmune diseases were included in the study.

We consider MAP infected individual the one that manifest a strong humoral response against MAP specific protein and/or the one that are positive to IS900 PCR. In fact, MAP infection is mainly asymptomatic, and the clinical symptoms (such as general malaise, chronic weight, abdominal pain and diarrhea) may manifest only after several years.

\section{SNP selection and genotyping}

Genomic DNA was purified from whole blood according to standard procedures [7]. Eight polymorphisms (rs2276631, rs3731865, rs3731864, rs17221959, rs2695342, rs2279015, rs17235409, rs17235416) were genotyped by PCR restriction fragment length polymorphism (RFLP) analysis, and the set of specific primers were designed based on the GenBank reference sequence (accession no. NT_005403). In detail, the following primer sequences were used: rs2276631 forward (5'TGCCACCATCCCTATACCCAG-3') and rs2276631 reverse ( 3'-TCTCGAAAGTGTCCCACTCAG-5'); rs3731865 forward (5'TCTCTGGCTGAAGGCTCTCC-3') and rs3731865 reverse (3'- TGTGCTATCAGTTGAGCCTC-5'); rs3731864 forward (5'CTGGACCAGGCTGGGCTGAC-3') and rs3731864 reverse (3'-CCACCACTCCCCTATGACGTG-5'); rs17221959 forward (5'CTTGTCCTGACCAGGCTCCT-3') and rs17221959 reverse (3'-CATGGCTCCGACTGAGTGAG-5'); rs2695342 forward (5'TCCTTGATCTTCGTAGTCTC-3') and rs2695342 reverse (3'- GGCTTACAGGACATGAGTAC-5'); rs2279015 forward (5'GCAAGTTGAGGAGCCAAGAC-3') and rs2279015 reverse (3'-ACCTGCATCAACTCCTCTTC-5'); rs17235409/rs17235416 forward (5'-
GCATCTCCCCAATTCATGGT-3') and rs 17235409/rs17235416 reverse (3'AACTGTCCCACTCTATCCTG-5') [8].

IS900 Map PCR

Detection of MAP DNA in 100 MS patients and $100 \mathrm{HCs}$ was performed as previously described [2]. Summing up, DNA was extracted from the PBMCs of both MS patients and HCs according to CTAB methodology. The presence of MAP specific DNA was detected by PCR amplification of IS900 sequences. Two rounds of amplifications were undertaken. First, primers LIZ 1 and LIZ 2, and secondly, primers AV1 and AV 2, were used to amplify a 298-bp fragment specific for MAP.

\section{MAP2694 cloning and expression}

MAP2694 was cloned and expressed as previously published [1].

\section{ELISA}

ELISA was performed as previously reported [2]. Briefly, ELISA plates were coated with $5 \mu \mathrm{g} / \mathrm{ml}$ of MAP2694 protein in carbonate bicarbonate buffer overnight at $4{ }^{\circ} \mathrm{C}$. The following day, the plates were blocked with $5 \%$ non-fat dried milk for one hour at room temperature. After that, 1:100 diluted sera samples were added for two hours. Finally, an alkaline phosphatase conjugated secondary antibody (Sigma-Aldrich) diluted 1:1000 was added for one hour and then the substrate was added. Plates were read at $405 \mathrm{~nm}$ on a VERSA Tunable Max Microplate Reader (Molecular Devices, Sunnyvale, CA, USA). Plates were washed between each incubation with $0.1 \%$ Tween-20 in PBS.

\section{Results}

Eight SLC11A1 SNPs were typed by PCR (RFLP) in $100 \mathrm{MS}$ patients and $100 \mathrm{HCs}$. Only rs 2276631 SNP was associated with MS. The genotype frequency for the allele AA was $40 \%$ in MS patients and $7 \%$ in the HCs $(\chi 2=28.5, p<0.0001)$. The frequency of A allele was $60 \%$ in MS patients and $38.5 \%$ in HCs $(\chi 2$ $=17.6, \mathrm{OR}=1.95, \mathrm{p}<0.0001)$. Both results were statistically significant (Table).

MAP DNA presence was detected by IS900 PCR in 23 out of $100 \mathrm{MS}$ patients $(23 \%)$ and in 7 out of $100 \mathrm{HCs}$ (7\%); the association was considered to be very statistically significant $(\chi 2=8, p=0.0023, O R=3.9)$. 
Table. Allelic and genotypic analyses of the associations between SLC11A1 polymorphisms and multiple sclerosis

\begin{tabular}{|c|c|c|c|c|c|c|c|c|c|}
\hline \multirow[t]{2}{*}{ SNP Identity } & \multicolumn{5}{|c|}{ Allele frequency } & \multicolumn{4}{|c|}{ Genotype frequency } \\
\hline & Location & A (\%) & a $(\%)$ & OR (CI 95\%) & p & AA $(\%)$ & Aa $(\%)$ & aa $(\%)$ & p \\
\hline rs2276631 & Exon 3 & & & & & & & & \\
\hline $\operatorname{MS}(n=100)$ & & $120(60 \%)$ & $80(40 \%)$ & $\mathbf{1 . 9 5}(1.31-2.91)$ & $<0.0001$ & $40(40 \%)$ & $40(40 \%)$ & $20(20 \%)$ & $<0.0001$ \\
\hline HCs $(n=100)$ & & $77(38.5 \%)$ & $123(61.5 \%)$ & & & $7(\%)$ & $63(63 \%)$ & $30(30 \%)$ & \\
\hline rs3731865 & Intron 4 & & & & & & & & \\
\hline $\operatorname{MS}(n=100)$ & & $135(67.5 \%)$ & $65(32.5 \%)$ & NS & NS & $46(46 \%)$ & $43(43 \%)$ & $11(11 \%)$ & NS \\
\hline HCs $(n=100)$ & & $133(66.5 \%)$ & $67(33.5 \%)$ & & & $42(42 \%)$ & $49(49 \%)$ & $9(9 \%)$ & \\
\hline rs3731864 & Intron 5 & & & & & & & & \\
\hline MS (n=100) & & $5(2.5 \%)$ & $195(97.5 \%)$ & NS & NS & $0(0 \%)$ & $5(5 \%)$ & $95(95 \%)$ & NS \\
\hline HCs $(n=100)$ & & $6(3 \%)$ & $194(97 \%)$ & & & $0(0 \%)$ & $6(6 \%)$ & $94(94 \%)$ & \\
\hline rs17221959 & Exon 8 & & & & & & & & \\
\hline MS (n=100) & & $97(48.5 \%)$ & $103(51.5 \%)$ & NS & NS & $19(19 \%)$ & $59(59 \%)$ & $22(22 \%)$ & NS \\
\hline HCs $(n=100)$ & & $86(43 \%)$ & $114(57 \%)$ & & & $10(10 \%)$ & $66(66 \%)$ & $24(24 \%)$ & \\
\hline rs2695342 & Exon 9 & & & & & & & & \\
\hline MS (n=100) & & $122(61 \%)$ & $78(39 \%)$ & NS & NS & $22(22 \%)$ & $78(78 \%)$ & $0(0 \%)$ & NS \\
\hline HCs $(n=100)$ & & $125(62.5 \%)$ & $75(37.5 \%)$ & & & $25(25 \%)$ & $75(75 \%)$ & $0(0 \%)$ & \\
\hline rs2279015 & Intron 13 & & & & & & & & \\
\hline MS $(n=100)$ & & $68(34 \%)$ & $132(66 \%)$ & NS & NS & $1(1 \%)$ & $66(66 \%)$ & $33(33 \%)$ & NS \\
\hline HCs $(n=100)$ & & $71(35.5 \%)$ & $129(64.5 \%)$ & & & $0(0 \%)$ & $71(71 \%)$ & $29(29 \%)$ & \\
\hline rs17235409 & Exon 15 & & & & & & & & \\
\hline $\mathrm{MS}(\mathrm{n}=100)$ & & $13(6.5 \%)$ & $187(93.5 \%)$ & NS & NS & $4(4 \%)$ & $5(5 \%)$ & $91(91 \%)$ & NS \\
\hline $\mathrm{HCs}(\mathrm{n}=100)$ & & $10(5 \%)$ & $190(95 \%)$ & & & $3(3 \%)$ & $4(4 \%)$ & $93(93 \%)$ & \\
\hline rs 17235416 & 3'UTR & & & & & & & & \\
\hline $\operatorname{MS}(\mathrm{n}=100)$ & & $5(5 \%)$ & $195(97.5 \%)$ & NS & NS & $0(0 \%)$ & $5(5 \%)$ & $95(95 \%)$ & NS \\
\hline HCs $(n=100)$ & & $3(3 \%)$ & $197(98.5 \%)$ & & & $0(0 \%)$ & $3(3 \%)$ & $97(97 \%)$ & \\
\hline
\end{tabular}

Single Nucleotide Polymorphism (SNP). Odds ratio (OR) was calculated at 95\% confidence intervals (CI); p value was calculated by Chi-square with Yates' correction and Fisher's exact test (the results was the same) 
In the same population study, antibodies against MAP2694 were searched. A strong humoral response was detected in $36 \%$ of MS patients and only in 3\% of $\mathrm{HCs}$, showing an association extremely statistically significant $(\chi 2=32, p<0.0001, \mathrm{OR}=18)$. Receiver operator characteristic $(\mathrm{ROC})$ curves analysis $(\mathrm{AUC}=$ 0.76) is shown in the Figure.

Moreover, binary logistic regression allowed us to observe a correlation between rs2276631 and MAP6994 sero-positivity $(\chi 2=39$; MAP2694 OR $=6$, $\mathrm{p}<0.0001 ; \mathrm{rs} 2276631 \mathrm{OR}=3, \mathrm{p}=0.008)$.

\section{Discussion}

The etiology of MS is thought to be caused by the joint action of genetic factors in the presence of other unidentified environmental agents. Several studies have suggested that different pathogens could trigger autoimmunity by molecular mimicry [9]. Counting one of the highest MS prevalence worldwide, Sardinia is an ideal setting for studying MS [3]. Genetic predisposition alone cannot account for such a high rate of MS, justifying the search for environmental agents capable of triggering the disease.

MAP is an obligate pathogenic bacterium, which was proven to play a role in several autoimmune diseases. Recently our group reported MAP involvement in MS [1,2]. The association was postulated in light of the strong humoral response directed against two MAP specific proteins (MAP2694, MAPFprB), and the detection of MAP's DNA in the peripheral blood of MS patients. To note, SLC11A1 polymorphisms have been previously linked with mycobacterial infections and autoimmune disease [10]. This gene exerts pleiotropic effects on macrophage function, regulating both cytokine production and antigen presentation. All of these activities are not only essential for protection against mycobacterial infection, but also critically involved in the induction and progression of autoimmune diseases.

In view of this observation, we wanted to investigate whether the SLC11A1 polymorphisms were associated with an altered susceptibility to MS; in particular we aimed to elucidate if there was an interaction between predisposing genes and MAP presence. The association of the HLA class II locus with MS and several novel susceptibility loci with modest effects have been recently identified [11]. The role of SLC11A1 in MS is controversial: on one hand, some studies deny an association with this pathology [12], while on the other hand, a significant association was reported between MS and the SLC11A1 gene [11].

At present we have PCR-RFLP typed $100 \mathrm{MS}$ patients and $100 \mathrm{HCs}$ for eight SLC11A1-SNPs. A larger sample size study has been initiated, which will

Figure. Receiver operator characteristic (ROC) curves analysis of anti-MAP2694 antibodies (Abs) in 100 MS patients and 100 healthy controls

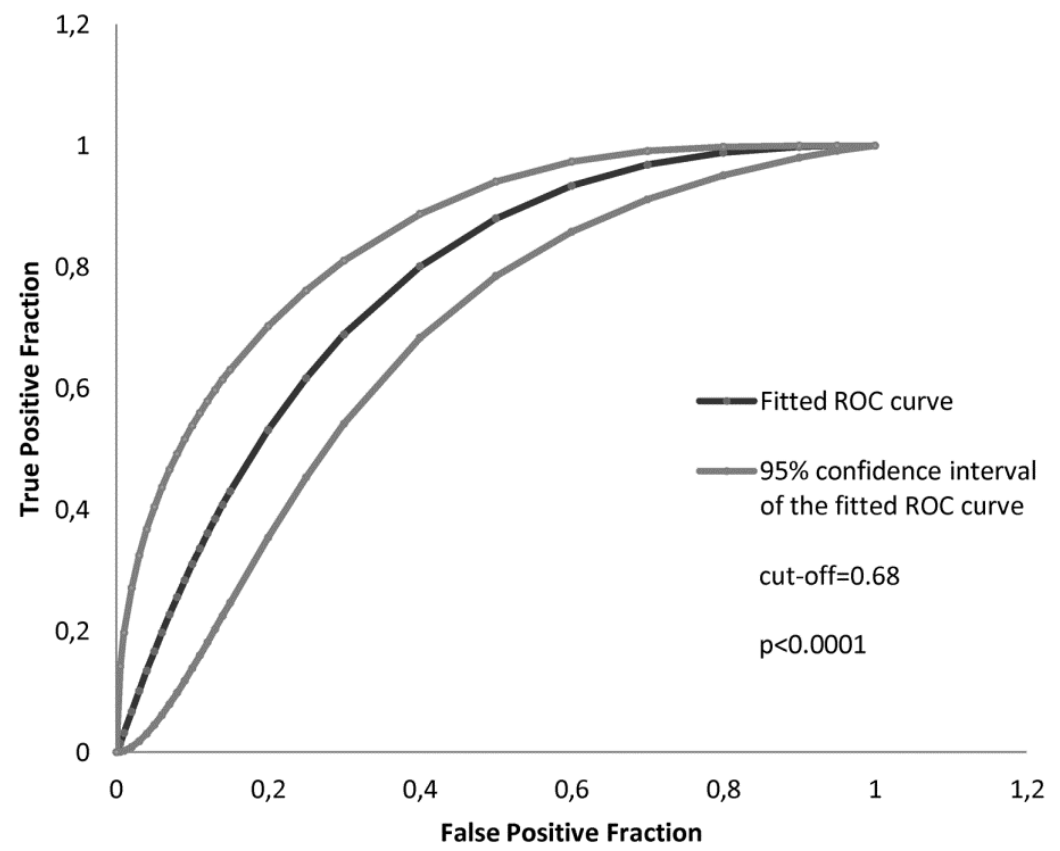

The cut-off was set at $>95 \%$ specificity calculated accordingly. Cut-off, area under ROC curve (AUC), and $p$ value, are given in the bottom right corner. 
look at more SNPs within this locus. The methodology will be improved as well, undertaking sequence analyses. To note, polymorphisms at the functional 5'(GT) n promoter region will be studied. The 5'(GT)n repeat polymorphism (rs34448891) has been previously associated with MS [13].

Presently we report a novel association between MS and the rs2276631 SNP within the SLC11A1 gene. The polymorphism rs2276631 is a nucleotide substitution that occurs in exon 3 (codon 66 for Phe). It consists of a $\mathrm{C} / \mathrm{T}$ substitution at residue 274 , which could impair iron transport through the channel. This in turn could avoid fagosome acidification and allow the mycobacterial survival inside the macrophage.

The presence of MAP DNA and a strong humoral response against MAP2694 antigen was also detected in MS Sardinian patients. Our preliminary results suggest that rs2276631 SNP might contribute to increased susceptibility to MS. Furthermore, a correlation between ELISA sero-positivity and SNP presence in $20 \%$ of the cases was observed. The association between rs2276631 SNP and Type 1 diabetes was recently reported [7], suggesting that the same polymorphism may be common to the major autoimmune diseases widespread in Sardinia.

\section{Conclusion}

Although this is an ongoing study, and it can be misleading to make premature conclusions, this first round of data suggests that the Sardinian population might be prone to develop autoimmune diseases due to polymorphisms in the SLC11A1 gene, which is important in the immune response against intracellular bacteria. Further studies are needed now to validate this report. MAP or another infectious agent could act as a trigger in igniting $\mathrm{MS}$ in genetically prone individuals.

\section{Acknowledgements}

This article is dedicated to our mentor, Prof Giovanni Fadda, a great teacher of science and life.

This study was partially supported by the Sardinian Region, L.R. 7, progetti di ricerca di base 2009.

\section{References}

1. Cossu D, Cocco E, Paccagnini D, Masala S, Ahmed N, Frau J, Marrosu MG, Sechi LA (2011) Association of Mycobacterium avium subsp. paratuberculosis with Multiple Sclerosis in Sardinian Patients. PLoS ONE 6: e18482.

2. Cossu D, Masala S, Cocco E, Paccagnini D, Frau J, Marrosu MG, Sechi LA (2012) Are Mycobacterium avium subsp. paratuberculosis and Epstein-Barr virus triggers of multiple sclerosis in Sardinia? Mult Scler 18: 1181-1184.
3. Cocco E, Sardu C, Massa R, Mamusa E, Musu L, Ferrigno P, Melis M, Montomoli P, Ferretti V, Coghe G, Fenu G, Frau J, Lorefice L, Carboni N, Contu P, Marrosu MG (2011) Epidemiology of multiple sclerosis in south-western Sardinia. Mult Scler 17: 1282-1289.

4. Blackwell JM, Searle S, Goswami T, Miller EN (2000) Understanding the multiple functions of NRAMP1. Microbes Infect 2: 317-321.

5. Sapkota BR, Hijikata M, Matsushita I, Tanaka G, Ieki R, Kobayashi N, Toyota E, Nagai H, Kurashima A, Tokunaga K, Keicho N (2012) Association of SLC11A1 (NRAMP1) polymorphisms with pulmonary Mycobacterium avium complex infection. Hum Immunol 73: 529-536.

6. Polman C, Reingold S, Branwell B, Clanet M, Cohen JA, Filippi M, Fujihara K, Havrdova E, Hutchinson M, Kappos L, Lublin FD, Montalban X, O'Connor P, Sandberg-Wollheim M, Thompson AJ, Waubant E, Weinshenker B, Wolinski JS (2011) Diagnostic criteria for multiple sclerosis: 2010 revisions to the McDonald criteria. Ann Neurol 69: 292-302.

7. Paccagnini D, Sieswerda L, Rosu V, Masala S, Pacifico A, Gazouli M, Ikonomopoulos J, Ahmed N, Zanetti S, Sechi LA (2009) Linking chronic infection and autoimmune diseases: Mycobacterium avium subsp. paratuberculosis, SLC11A1 polymorphisms and type-1 diabetes mellitus. PLoS One 4: e7109.

8. Liu J, Fujiwara TM, Buu NT, Sanchez FO, Cellier M, Paradis AJ, Frappier D, Skamene E, Gros P, Morgan K, Schurr E (1995) Identification of Polymorphisms and Sequence Variants in the Human Homologue of the Mouse Natural Resistance-Associated Macrophage Protein Gene. Am J Hum Genet 56: 845-853.

9. Oldstone MB (2005) Molecular mimicry, microbial infection, and autoimmune disease: evolution of the concept. Curr Top Microbiol Immunol. 296: 1-17.

10. Dai YD, Marrero IG, Gros P, Zaghouani H, Wicker LS, Sercarz EE (2009) Slc1lal enhances the autoimmune diabetogenic T-cell response by altering processing and presentation of pancreatic islet antigens. Diabetes 1: 156-164.

11. The International Multiple Sclerosis Genetics Consortium \& the Wellcome Trust Case Control Consortium 2 (2011) Genetic risk and a primary role for cell-mediated immune mechanisms in multiple sclerosis. Nature 476: 214-219.

12. Ates O, Kurt S, Bozkurt N, Karaer H (2010) NRAMP1 (SLC11A1) variants: genetic susceptibility to multiple Sclerosis. J Clin Immunol 30: 583-586.

13. Gazouli M, Sechi L, Paccagnini D, Sotgiu S, Arru G, Nasioulas G, Vassilopoulos D (2008) NRAMP1 polymorphism and viral factors in Sardinian multiple sclerosis patients. Can J Neurol Sci 35: 491-494.

\section{Corresponding author}

Professor Leonardo Antonio Sechi

Dipartimento di Scienze Biomediche

Sezione di Microbiologia Sperimentale e Clinica

Università di Sassari, Italy

Viale San Pietro 43B

Sassari Italy

Telephone/Fax:+39-079212345

Emailsechila@uniss.it

Conflict of interests: No conflict of interests is declared. 